\title{
Multiplicity results for a generalized Sturm- Liouville dynamical system on time scales
}

Youwei Zhang

Correspondence: ywzhang0288@163.com Department of Mathematics, Hexi University, Gansu 734000, People's Republic of China

\section{Abstract}

By applying the fixed point theorem in cones, some new and general results on the existence of positive solution to second order generalized Sturm-Liouville dynamical system on time scale $\mathbb{T}$

$$
\left\{\begin{array}{l}
u_{1}^{\Delta \Delta}(t)+h_{1}(t) f_{1}\left(t, u_{1}(t), u_{2}(t), u_{1}^{\Delta}(t), u_{2}^{\Delta}(t)\right)=0, \\
u_{2}^{\Delta \Delta}(t)+h_{2}(t) f_{2}\left(t, u_{1}(t), u_{2}(t), u_{1}^{\Delta}(t), u_{2}^{\Delta}(t)\right)=0, \\
a u_{i}\left(t_{1}\right)-b u_{i}^{\Delta}\left(t_{1}\right)=\sum_{k=1}^{m-2} a_{k} u_{i}\left(\xi_{k}\right), \quad(i=1,2) \\
c u_{i}\left(\sigma\left(t_{2}\right)\right)+d u_{i}^{\Delta}\left(\sigma\left(t_{2}\right)\right)=\sum_{k=1}^{m-2} b_{k} u_{i}\left(\xi_{k}\right),
\end{array}(i=1,\right.
$$

are obtained. The first-order $\Delta$-derivatives are involved in the nonlinear terms explicitly.

Mathematics Subject Classification (2000) 39A10

Keywords: Sturm-Liouville dynamical system, Cone, Fixed point, Positive solution, Time scales

\section{Introduction}

Time scales theory allows us to handle systematically the continuous and discrete problems and to setup a certain structure, which is to play the role of generalizing continuous and discrete $[1,2]$, as well as more general systems simultaneously. The study of time scales has led to several important applications, for example, in the study of insect population models, phytoremediation of metals, wound healing, and epidemic models [3-5].

Dynamic systems have been the subject of numerous investigations and are also used of various real processes and phenomena in physics, such as, dynamics of gas flow, Newtonian fluid mechanics, nuclear physics, boundary layer theory, and so on, which during their evolutionary processes experience an abrupt change of state at certain moments of time, we refer to Wang [6], Zhang [7] and the references therein. Recently, there have seen a significant development and have made substantial progress on discussing the solutions for the dynamic systems on time scales, the main research theories used is the fixed point theorems in cones. Furthermore, noting an operation on the space of functions from one state space to other state space has to be defined, generalizing the continuous and discrete operations [8]. Applying the operation on the space of functions to discuss the solutions for the dynamical systems on time scales, it can bridge the gap between the continuous and discrete, mainly, can avoid the respective study in the solutions of

\section{SpringerOpen ${ }^{\circ}$}

(C) 2011 Zhang; licensee Springer. This is an Open Access article distributed under the terms of the Creative Commons Attribution License (http://creativecommons.org/licenses/by/2.0), which permits unrestricted use, distribution, and reproduction in any medium, provided the original work is properly cited. 
continuous and discrete systems. That is to say, the field of dynamic systems on time scales contains and extends the classical dynamical systems for differential and difference. For the related results, we refer to [9-12]. Specially, the first-order derivatives are involved in the nonlinear terms explicitly in dynamic system, which describe more complicated phenomena and possess some value of applications. But very little work has been done on the existence of solutions to this variety of dynamical systems on time scales. Based on the known results of boundary value problems for the differential and difference equation, the lower order derivatives are involved in the nonlinear terms explicitly. This study is devoted to proving the existence of solutions for a generalized Sturm-Liouville dynamical system on time scales $\mathbb{T}$

$$
\left\{\begin{array}{l}
u_{1}^{\Delta \Delta}(t)+h_{1}(t) f_{1}\left(t, u_{1}(t), u_{2}(t), u_{1}^{\Delta}(t), u_{2}^{\Delta}(t)\right)=0, \\
u_{2}^{\Delta \Delta}(t)+h_{2}(t) f_{2}\left(t, u_{1}(t), u_{2}(t), u_{1}^{\Delta}(t), u_{2}^{\Delta}(t)\right)=0, \\
a u_{i}\left(t_{1}\right)-b u_{i}^{\Delta}\left(t_{1}\right)=\sum_{k=1}^{m-2} a_{k} u_{i}\left(\xi_{k}\right), \quad(i=1,2), \\
c u_{i}\left(\sigma\left(t_{2}\right)\right)+d u_{i}^{\Delta}\left(\sigma\left(t_{2}\right)\right)=\sum_{k=1}^{m-2} b_{k} u_{i}\left(\xi_{k}\right),
\end{array} \quad\left(t_{1}, t_{2}\right]_{\mathbb{T}},\right.
$$

where $\sigma(\cdot)$ is the forward jump operator, $a, b, c, d, a_{k}, b_{k}, \xi_{k}, h_{i}$ and $f_{i}(i=1,2)$ satisfy $\left(A_{1}\right) a, b, c, d \in[0, \infty), a_{k}, b_{k} \in(0, \infty)(k=1,2, \ldots, m-2), a>\sum_{k=1}^{m-2} a_{k}, c>\sum_{k=1}^{m-2} b_{k}$, $t_{1}<\xi_{1}<\ldots<\xi_{m-2}<t_{2}$

$\left(A_{2}\right) h_{i} \in C_{r d}\left(\left[t_{1}, t_{2}\right]_{\mathbb{T}},[0, \infty)\right)(i=1,2)$ and there exists $t_{0} \in\left(t_{1}, t_{1}\right)_{\mathbb{T}}$ such that $h_{i}\left(t_{0}\right)>$ $0, f_{i} \in C\left(\left[t_{1}, t_{2}\right]_{\mathbb{T}} \times[0, \infty)^{2} \times(-\infty, \infty)^{2},[0, \infty)\right)(i=1,2)$.

A solution $u(t):=\left(u_{1}(t), u_{2}(t)\right)$ of the system $(S)$ is positive if, for each $i=1,2, u_{i}(t) \geq$ 0 for all $t \in\left[t_{1}, t_{2}\right]_{\mathbb{T}}$, and there is at least one nontrivial component of $u(t)$.

We would like to mention some results of Li and Sun [9], which motivated us to consider our problem $(S)$. In [9], the authors have studied the existence criteria of at least triple nonnegative solutions for a dynamical system on a measure chain

$$
\left\{\begin{array}{c}
u_{1}^{\Delta \Delta}(t)+f_{1}\left(t, u_{1}(\sigma(t)), u_{2}(\sigma(t))\right)=0, \\
u_{2}^{\Delta \Delta}(t)+f_{2}\left(t, u_{1}(\sigma(t)), u_{2}(\sigma(t))\right)=0, \\
\alpha u_{i}(a)-\beta u_{i}^{\Delta}(a)=0, \quad \gamma u_{i}(\sigma(b))+\delta u_{i}^{\Delta}(\sigma(b))=0(i=1,2),
\end{array}\right.
$$

where $\alpha, \beta, \gamma, \delta \geq 0, f_{i}:[a, b] \times[0, \infty) \times[0, \infty) \rightarrow[0, \infty)$ is continuous, the main tools used is the fixed point theorems in cones. In addition, Sun et al. [11] considered a discrete system with parameter

$$
\left\{\begin{array}{l}
\Delta^{2} u_{i}(k)+\lambda h_{i}(k) f_{i}\left(u_{1}(k), u_{2}(k), \ldots, u_{n}(k)\right)=0, \quad k \in[0, T] \\
u_{i}(0)=u_{i}(T+2)=0 \quad(i=1,2, \ldots, n)
\end{array}\right.
$$

where $\lambda>0$ is a constant, $T$ and $n \geq 2$ are two fixed positive integers. They have established the existence of one positive solution by using the theory of fixed point index. Inspired by $[6,7,9,13,14]$ et al, this study establishes some new and more general results for the existence of multiple positive solutions to the dynamical system $(S)$. The results are even new for the general time scales as well as in special case of the continuous and discrete dynamical systems. Our results extend the known results of $\mathrm{Li}$ and Sun [9] $\left(a_{k}=0, b_{k}=0(k=1,2, \ldots, m-2)\right)$ from another point of view, our Theorem 3.2 improve the main results of Shao and Zhang [12]. Specially, taking $\mathbb{T}=\mathbb{R}$, our 
Theorem 3.2 extend the main results of Zhang and Liu [7]; taking $\mathbb{T}=\mathbb{Z}$, our Theorem 3.3 improve the main results of Sun et al. [11].

The rest of the paper is organized as follows. Section 2 provides some background material for discussing the generalized Sturm-Liouville dynamical system $(S)$. An important lemma and a criterion for the existence of three positive solutions to the dynamical system $(S)$ are established, the results are tested on an example, and a general result for dynamical system is considered in Section 3.

\section{Preliminaries}

Let $\gamma$ and $\theta$ be nonnegative continuous convex functionals on a cone $P, \alpha$ be a nonnegative continuous concave functional on $P, \beta$ be a nonnegative continuous functional on $P$, and $m_{1}, m_{2}, m_{3}$ and $m_{4}$ be positive numbers. We define the following convex sets

$$
\begin{gathered}
P\left(\gamma, m_{4}\right)=\left\{u \in P: \gamma(u)<m_{4}\right\}, \\
P\left(\gamma, \alpha, m_{2}, m_{4}\right)=\left\{u \in P: m_{2} \leq \alpha(u), \gamma(u) \leq m_{4}\right\}, \\
P\left(\gamma, \theta, \alpha, m_{2}, m_{3}, m_{4}\right)=\left\{u \in P: m_{2} \leq \alpha(u), \theta(u) \leq m_{3}, \gamma(u) \leq m_{4}\right\},
\end{gathered}
$$

and a closed set

$$
Q\left(\gamma, \beta, m_{1}, m_{4}\right)=\left\{u \in P: m_{1} \leq \beta(u), \gamma(u) \leq m_{4}\right\} .
$$

To prove our main results, we state the Avery and Peterson fixed point theorem [15].

Lemma 2.1 Let $P$ be a cone in a real Banach space $\mathcal{B}$. Let $\gamma$ and $\theta$ be nonnegative continuous convex functionals on $P, \alpha$ be a nonnegative continuous concave functional on $P$, and $\beta$ be a nonnegative continuous functional on $P$ satisfying $\beta(\lambda u) \leq \lambda \beta(u)$ for 0 $\leq \lambda \leq 1$, such that for some positive numbers $\varepsilon$ and $m_{4}$,

$$
\alpha(u) \leq \beta(u) \text { and }\|u\| \leq \varepsilon \gamma(u) \text { for all } u \in \overline{P\left(\gamma, m_{4}\right)} .
$$

Suppose $T: \overline{P\left(\gamma, m_{4}\right)} \rightarrow \overline{P\left(\gamma, m_{4}\right)}$ is completely continuous and there are positive numbers $m_{1}, m_{2}$ and $m_{3}$ with $m_{1}<m_{2}$ such that

$\left(B_{1}\right)\left\{u \in P\left(\gamma, \theta, \alpha, m_{2}, m_{3}, m_{4}\right): \alpha(u)>m_{2}\right\} \neq \varnothing, \alpha(T u)>m_{2}$ for $u \in P\left(\gamma, \theta, \alpha, m_{2}\right.$, $\left.m_{3}, m_{4}\right)$;

$\left(B_{2}\right) \alpha(T u)>m_{2}$ for $u \in P\left(\gamma, \alpha, m_{2}, m_{4}\right)$ with $\theta(T u)>m_{3}$;

$\left(B_{3}\right) 0 \notin Q\left(\gamma, \beta, m_{1}, m_{4}\right)$ and $\beta(T u)<m_{1}$ for $u \in Q\left(\gamma, \beta, m_{1}, m_{4}\right)$ with $\beta(u)=m_{1}$.

Then $T$ has at least three fixed points $u_{\mathbb{1}}, u_{\mathfrak{Z}}, u_{\Im} \in \overline{P\left(\gamma, m_{4}\right)}$ such that

$$
\begin{gathered}
\quad \gamma\left(u_{j}\right) \leq m_{4}, \quad(j=\mathbb{1}, 2, \Re) ; \\
m_{2}<\alpha\left(u_{\mathbb{1}}\right) ; \quad m_{1}<\beta\left(u_{2}\right) \text { with } \alpha\left(u_{2}\right)<m_{2} ; \quad \beta\left(u_{\Re}\right)<m_{1} .
\end{gathered}
$$

In order to discuss conditions for the existence of at least three positive solutions to the generalized Sturm-Liouville dynamical system $(S)$, we firstly define $x(t)=a\left(t-t_{1}\right)+$ $b, y(t)=c\left(\sigma\left(t_{2}\right)-t\right)+d, t \in\left[t_{1}, \sigma\left(t_{2}\right)\right]_{\mathbb{T}}$. It is easy to see that $x(t)$ and $y(t)$ are solutions of the problem $-x^{\Delta \Delta}(t)=0, x\left(t_{1}\right)=b, x^{\Delta}\left(t_{1}\right)=a$ and $-y^{\Delta \Delta}(t)=0, y\left(\sigma\left(t_{2}\right)\right)=d, y^{\Delta}\left(\sigma\left(t_{2}\right)\right)$ $=-c$, respectively. Let $G(t, s)$ be Green's function of the boundary value problem

$$
\left\{\begin{array}{l}
u^{\Delta \Delta}(t)=0, \quad t \in\left[t_{1}, t_{2}\right]_{\mathbb{\pi}} \\
a u\left(t_{1}\right)-b u^{\Delta}\left(t_{1}\right)=0, c u\left(\sigma\left(t_{2}\right)\right)+d u^{\Delta}\left(\sigma\left(t_{2}\right)\right)=0 .
\end{array}\right.
$$


It is known [16] that

$$
G(t, s)=\frac{1}{r} \begin{cases}x(t) y(\sigma(s)), & t \leq s, \\ x(\sigma(s)) y(t), & \sigma(s) \leq t,\end{cases}
$$

for $t \in\left[t_{1}, \sigma\left(t_{2}\right)\right]_{\mathbb{T}}$ and $s \in\left[t_{1}, t_{2}\right]_{\mathbb{T}}$, where $r=b c+a d+a c\left(\sigma\left(t_{2}\right)-t_{1}\right)$.

Define

$$
\eta=\min \left\{t \in \mathbb{T}: t \geq \frac{3 t_{1}+\sigma\left(t_{2}\right)}{4}\right\}, \quad \omega=\max \left\{t \in \mathbb{T}: t \leq \frac{t_{1}+3 \sigma\left(t_{2}\right)}{4}\right\} .
$$

Obviously, $\eta$ and $\omega$ exist, and satisfy

$$
\frac{3 t_{1}+\sigma\left(t_{2}\right)}{4} \leq \eta<\omega \leq \frac{t_{1}+3 \sigma\left(t_{2}\right)}{4} .
$$

Further,

$$
\text { if } \sigma(\omega)=\mathrm{t}_{2} \text { and } d=0 \text {, then } t_{2}<\sigma\left(t_{2}\right) .
$$

For the Green's function $G(t, s)$, we have

$$
0 \leq G(t, s) \leq G(\sigma(s), s), \quad(t, s) \in\left[t_{1}, \sigma\left(t_{2}\right)\right]_{\mathbb{}} \times\left[t_{1}, t_{2}\right]_{\pi},
$$

since

$$
\frac{G(t, s)}{G(\sigma(s), s)}= \begin{cases}\frac{x(t)}{x(\sigma(s))}, & t \leq s \\ \frac{y(t)}{y(\sigma(s))}, & \sigma(s) \leq t .\end{cases}
$$

Furthermore, we obtain

$$
G(t, s) \geq \bar{k} G(\sigma(s), s),
$$

Where $\bar{k}=\min _{(s, t) \in\left[t_{1}, \sigma\left(t_{2}\right)\right]_{\mathbb{T}} \times}\left[\frac{3 t_{1}+\sigma\left(t_{2}\right)}{4}, \frac{t_{1}+3 \sigma\left(t_{2}\right)}{4}\right]_{\mathbb{T}} \frac{G(t, s)}{G(\sigma(s), s)}$,

$$
G(t, s) \geq k G(\sigma(s), s)
$$

Where $k=\min _{(s, t) \in\left[t_{1}, \sigma\left(t_{2}\right)\right]_{\mathbb{T}} \times[\eta, \sigma(\omega)]_{\mathbb{T}}} \frac{G(t, s)}{G(\sigma(s), s)}$. Obviously, $0<\bar{k} \leq k<1$.

Denote

$$
E=\left|\begin{array}{ll}
-\sum_{k=1}^{m-2} a_{k} x\left(\xi_{k}\right) & r-\sum_{k=1}^{m-2} a_{k} \gamma\left(\xi_{k}\right) \\
r-\sum_{k=1}^{m-2} b_{k} x\left(\xi_{k}\right) & -\sum_{k=1}^{m-2} b_{k} \gamma\left(\xi_{k}\right)
\end{array}\right| .
$$

Lemma 2.2 Assume $\left(A_{1}\right)$ holds. If $E \neq 0$, then for $g_{i} \in C_{r d}\left(\left[t_{1}, t_{2}\right]_{\mathbb{T}}\right)(i=1,2)$, the system

$$
\left\{\begin{array}{l}
u_{1}^{\Delta \Delta}(t)+g_{1}(t)=0, \\
u_{2}^{\Delta \Delta}(t)+g_{2}(t)=0, t \in\left[t_{1}, t_{2}\right]_{\mathbb{T}}, \\
a u_{i}\left(t_{1}\right)-b u_{i}^{\Delta}\left(t_{1}\right)=\sum_{k=1}^{m-2} a_{k} u_{i}\left(\xi_{k}\right), \\
c u_{i}\left(\sigma\left(t_{2}\right)\right)+d u_{i}^{\Delta}\left(\sigma\left(t_{2}\right)\right)=\sum_{k=1}^{m-2} b_{k} u_{i}\left(\xi_{k}\right),
\end{array}(i=1,2)\right.
$$


has a unique solution

$$
\begin{aligned}
u(t)=\left(u_{1}(t), u_{2}(t)\right)= & \left(\int_{t_{1}}^{t_{2}} G(t, s) g_{1}(s) \Delta s+x(t) A\left(g_{1}\right)+y(t) B\left(g_{1}\right),\right. \\
& \left.\int_{t_{1}}^{t_{2}} G(t, s) g_{2}(s) \Delta s+x(t) A\left(g_{2}\right)+\gamma(t) B\left(g_{2}\right)\right),
\end{aligned}
$$

where

$$
\begin{aligned}
& A\left(g_{i}\right)=\frac{1}{E}\left|\begin{array}{lll}
\sum_{k=1}^{m-2} a_{k} \int_{t_{1}}^{t_{2}} G\left(\xi_{k}, s\right) g_{i}(s) \Delta s & r-\sum_{k=1}^{m-2} a_{k} y\left(\xi_{k}\right) \\
\sum_{k=1}^{m-2} b_{k} \int_{t_{1}}^{t_{2}} G\left(\xi_{k}, s\right) g_{i}(s) \Delta s & -\sum_{k=1}^{m-2} b_{k} y\left(\xi_{k}\right)
\end{array}\right|, \\
& B\left(g_{i}\right)=\frac{1}{E}\left|\begin{array}{lll}
-\sum_{k=1}^{m-2} a_{k} x\left(\xi_{k}\right) & \sum_{k=1}^{m-2} a_{k} \int_{t_{1}}^{\sigma\left(t_{2}\right)} G\left(\xi_{k}, s\right) g_{i}(s) \Delta s \\
r-\sum_{k=1}^{m-2} b_{k} x\left(\xi_{k}\right) & \sum_{k=1}^{m-2} b_{k} \int_{t_{1}}^{\sigma\left(t_{2}\right)} G\left(\xi_{k}, s\right) g_{i}(s) \Delta s
\end{array}\right| \quad(i=1,2) .
\end{aligned}
$$

Proof. The proof is similar to Lemma 2.2 [17], we omit it. $\square$ In addition, we give a necessary hypothesis:

$$
\left(A_{3}\right) E<0, r-\sum_{k=1}^{m-2} a_{k} y\left(\xi_{k}\right)>0, r-\sum_{k=1}^{m-2} b_{k} x\left(\xi_{k}\right)>0 .
$$

Lemma 2.3 If $\left(A_{1}\right)$ and $\left(A_{3}\right)$ hold, then for $g_{i} \in C_{r d}\left(\left[t_{1}, t_{2}\right]_{\mathbb{T}}\right)$ with $g_{i} \geq 0(i=1,2)$, there exists $\tau_{1} \in(0,1)$ such that the unique solution $u=\left(u_{1}, u_{2}\right)$ of the system (2.3) satisfies

$$
\begin{gathered}
u_{i}(t) \geq 0(i=1,2), \quad t \in\left[t_{1}, \sigma\left(t_{2}\right)\right]_{\mathbb{\Psi}} \\
\inf _{t \in[\eta, \sigma(\omega)]_{\mathbb{T}}} u_{1}(t)+\inf _{t \in[\eta, \sigma(\omega)]_{\mathbb{T}}} u_{2}(t) \geq \tau_{1}\left(\sup _{t \in\left[t_{1}, \sigma\left(t_{2}\right)\right]_{\mathbb{T}}}\left|u_{1}(t)\right|+\sup _{t \in\left[t_{1}, \sigma\left(t_{2}\right)\right]_{\mathbb{T}}}\left|u_{2}(t)\right|\right) .
\end{gathered}
$$

Proof. The proof is similar to Lemma 2.2 [18], we omit it. $\square$

\section{Main results}

$$
\begin{aligned}
& \text { Let } E=C_{r d}^{1}\left[t_{1}, \sigma\left(t_{2}\right)\right]_{\mathbb{T}} \quad \text { with the sup norm. Set } \\
& \|u\|_{0}=\sup _{t \in\left[t_{1}, \sigma\left(t_{2}\right)\right]_{\top}}\left|u_{1}(t)\right|+\sup _{t \in\left[t_{1}, \sigma\left(t_{2}\right)\right]_{\top}}\left|u_{2}(t)\right|,\left\|u^{\Delta}\right\|_{0}=\sup _{t \in\left[t_{1}, t_{2}\right]_{\top}}\left|u_{1}^{\Delta}(t)\right|+\sup _{t \in\left[t_{1}, t_{2}\right]_{\top}}\left|u_{2}^{\Delta}(t)\right|, \quad \text { Let } \\
& \mathcal{B}=E \times E \text {, the norm of } u \in \mathcal{B} \text { is defined by } \\
& \|u\|=\max \left\{\|u\|_{0},\left\|u^{\Delta}\right\|_{0}\right\},
\end{aligned}
$$

then $\mathcal{B}$ is a Banach space. The cone $P \subset \mathcal{B}$ is defined by

$$
P=\left\{\begin{array}{l}
u=\left(u_{1}, u_{2} \in \mathcal{B}\right): u_{i}(t) \text { is nonnegative and concave on }\left[t_{1}, \sigma\left(t_{2}\right)\right]_{\pi}, \\
u_{i} \text { satisfies } a u_{i}\left(t_{1}\right)-b u_{i}^{\Delta}\left(t_{1}\right)=\sum_{k=2}^{m-2} a_{k} u_{i}\left(\xi_{k}\right) \\
c u_{i}\left(\sigma\left(t_{2}\right)\right)+d u_{i}^{\Delta}\left(\sigma\left(t_{2}\right)\right)=\sum_{k=2}^{m-2} b_{k} u_{i}\left(\xi_{k}\right) \quad(i=1,2) \\
\quad \inf _{t \in[\eta, \sigma(\omega)]_{\pi}} u_{1}(t)+\inf _{t \in[\eta, \sigma(\omega)]_{\pi}} u_{2}(t) \\
\quad \geq \tau_{1}\left(\sup _{t \in\left[t_{1}, \sigma\left(t_{2}\right)\right]_{\pi}}\left|u_{1}(t)\right|+\sup _{t \in\left[t_{1}, \sigma\left(t_{2}\right)\right]_{\pi}}\left|u_{2}(t)\right|\right.
\end{array}\right\}
$$

Let the nonnegative continuous concave functional $\alpha$, the nonnegative continuous convex functionals $\beta$ and $\gamma$, and the nonnegative continuous functional $\theta$ be defined on the cone $P$ by 


$$
\begin{gathered}
\alpha(u)=\inf _{t \in[\eta \sigma(\omega)]_{\mathbb{T}}}\left|u_{1}(t)\right|+\inf _{t \in[\eta \sigma(\omega)]_{\mathbb{T}}}\left|u_{2}(t)\right|, \quad \gamma(u)=\sup _{t \in\left[t_{1}, t_{2}\right]_{\mathbb{T}}}\left|u_{1}^{\Delta}(t)\right|+\sup _{t \in\left[t_{1}, t_{2}\right]_{\mathbb{T}}}\left|u_{2}^{\Delta}(t)\right|, \\
\theta(u)=\beta(u)=\sup _{t \in\left[t_{1}, \sigma\left(t_{2}\right)\right]_{\top}}\left|u_{1}(t)\right|+\sup _{t \in\left[t_{1}, \sigma\left(t_{2}\right)\right]_{\top}}\left|u_{2}(t)\right| .
\end{gathered}
$$

Since the first-order $\Delta$-derivatives are involved in the nonlinear terms explicitly to the system $(S)$, we give the following lemma.

Lemma 3.1 Assume $\left(A_{1}\right)$ holds. If $u=\left(u_{1}, u_{2}\right) \in P$, then

$$
\|u\|_{0} \leq \tau_{2}\left\|u^{\Delta}\right\|_{0}
$$

where

$$
\tau_{2}=\max \left\{\frac{a\left(t_{0}-t_{1}\right)+b}{a-\sum_{k=1}^{m-2} a_{k}}, \frac{c\left(\sigma\left(t_{2}\right)-t_{0}\right)+d}{c-\sum_{k=1}^{m-2} b_{k}}\right\} .
$$

Proof. In fact, it stuffices to prove that

$$
\sup _{t \in\left[t_{1}, \sigma\left(t_{2}\right)\right]_{\pi}}\left|u_{1}(t)\right|+\sup _{t \in\left[t_{1}, \sigma\left(t_{2}\right)\right]_{\pi}}\left|u_{2}(t)\right| \leq \tau_{2}\left(\sup _{t \in\left[t_{1}, t_{2}\right]_{\pi}}\left|u_{1}^{\Delta}(t)\right|+\sup _{t \in\left[t_{1}, t_{2}\right]_{\pi}}\left|u_{2}^{\Delta}(t)\right|\right)
$$

holds. Suppose to contrary, there exists $t_{0}$ such that $u_{1}\left(t_{0}\right)+u_{2}\left(t_{0}\right)>\tau_{2}\left(\left|u_{1}^{\Delta}\left(t_{0}\right)\right|+\left|u_{2}^{\Delta}\left(t_{0}\right)\right|\right)$. Since $u=\left(u_{1}, u_{2}\right) \in P$, we choose that $D$ such that $D \subseteq\left[t_{1}, t_{2}\right]_{\mathbb{W}}^{\kappa}$, and $\left[t_{1}, t_{2}\right]_{\mathbb{W}}^{\kappa} \backslash D$ is countable and contains no right-scattered elements of $\left[t_{1}, t_{2}\right]_{\mathbb{1}}, u_{i}(t)(i=1,2)$ is continuous on $\left[t_{1}, t_{2}\right]_{\mathbb{T}}$ and differentiable in each $t \in D$, so $u_{i}(t)(i$ $=1,2)$ is pre-differentiable with $D$. Let $t_{0} \in \mathbb{T}$ such that $t_{1}<t_{0}<t_{2}$, and the compact intervals $U_{1}, U_{2} \subset\left[t_{1}, t_{2}\right]_{\rrbracket}$ such that $U_{1}$ has endpoints $t_{1}, t_{0}, U_{2}$ has endpoints $t_{0}, \sigma\left(t_{2}\right)$.

For the endpoint $t_{1}$, from the mean value theorem on time scales [1, p25], one has that

$$
\sup _{t \in U_{1}^{\wedge} \cap D}\left|u_{i}^{\Delta}(t)\right| \geq \frac{\left|u_{i}\left(t_{0}\right)-u_{i}\left(t_{1}\right)\right|}{\left|t_{0}-t_{1}\right|}=\frac{u_{i}\left(t_{0}\right)-\frac{1}{a}\left(b u_{i}^{\Delta}\left(t_{1}\right)+\sum_{k=1}^{m-2} a_{k} u_{i}\left(\xi_{i}\right)\right)}{t_{0}-t_{1}} \quad(i=1,2),
$$

$u_{i}(t) \quad(i=1,2)$ is concave on $U_{1}^{\kappa} \cap D$, it follows that $u_{i}^{\Delta}\left(t_{1}\right)=\sup _{t \in U_{1}^{k} \cap D}\left|u_{i}^{\Delta}(t)\right|(i=1,2)$.

We have

$$
\sup _{t \in U_{1}^{\kappa} \cap D}\left|u_{i}^{\Delta}(t)\right| \geq \frac{a-\sum_{k=1}^{m-2} a_{k}}{a\left(t_{0}-t_{1}\right)+b} u_{i}\left(t_{0}\right) \quad(i=1,2),
$$

so we have

$$
\sup _{t \in U_{1}^{\kappa} \cap D}\left|u_{1}^{\Delta}(t)\right|+\sup _{t \in U_{1}^{\kappa} \cap D}\left|u_{2}^{\Delta}(t)\right| \geq \frac{a-\sum_{k=1}^{m-2} a_{k}}{a\left(t_{0}-t_{1}\right)+b}\left(u_{1}\left(t_{0}\right)+u_{2}\left(t_{0}\right)\right) .
$$

For the endpoint $\sigma\left(t_{2}\right)$, one has that

$$
\begin{aligned}
\sup _{t \in U_{2}^{U} \cap D}\left|u_{i}^{\Delta}(t)\right| & \geq \frac{\left|u_{i}\left(\sigma\left(t_{2}\right)\right)-u_{i}\left(t_{0}\right)\right|}{\left|\sigma\left(t_{2}\right)-t_{0}\right|}=\frac{u_{i}\left(t_{0}\right)-u_{i}\left(\sigma\left(t_{2}\right)\right)}{\sigma\left(t_{2}\right)-t_{0}} \\
& =\frac{u_{i}\left(t_{0}\right)-\frac{1}{c}\left(-d u_{i}^{\Delta}\left(\sigma\left(t_{2}\right)\right)+\sum_{k=1}^{m-2} b_{k} u_{i}\left(\xi_{i}\right)\right)}{\sigma\left(t_{2}\right)-t_{0}} \quad(i=1,2),
\end{aligned}
$$

again, $u_{i}(t) \quad(i=1,2)$ is concave on $U_{1}^{\kappa} \cap D$, it follows that $u_{i}^{\Delta}\left(\sigma\left(t_{2}\right)\right)=-\sup _{t \in U_{2}^{k} \cap D}\left|u_{i}^{\Delta}(t)\right|(i=1,2)$. We have 


$$
\sup _{t \in U_{2}^{\kappa} \cap D}\left|u_{i}^{\Delta}(t)\right| \geq \frac{c-\sum_{k=1}^{m-2} b_{k}}{c\left(\sigma\left(t_{2}\right)-t_{0}\right)+d} u_{i}\left(t_{0}\right) \quad(i=1,2),
$$

we get

$$
\sup _{t \in U_{2}^{\kappa} \cap D}\left|u_{1}^{\Delta}(t)\right|+\sup _{t \in U_{2}^{\kappa} \cap D}\left|u_{2}^{\Delta}(t)\right| \geq \frac{c-\sum_{k=1}^{m-2} b_{k}}{c\left(\sigma\left(t_{2}\right)-t_{0}\right)+d}\left(u_{1}\left(t_{0}\right)+u_{2}\left(t_{0}\right)\right) .
$$

From the discussion results of the above cases, we can obtain that for $t=t_{0}$,

$$
\begin{aligned}
\left|u_{1}^{\Delta}\left(t_{0}\right)\right|+\left|u_{2}^{\Delta}\left(t_{0}\right)\right| & \geq \min \left\{\frac{a-\sum_{k=1}^{m-2} a_{k}}{a\left(t_{0}-t_{1}\right)+b}, \frac{c-\sum_{k=1}^{m-2} b_{k}}{c\left(\sigma\left(t_{2}\right)-t_{0}\right)+d}\right\}\left(u_{1}\left(t_{0}\right)+u_{2}\left(t_{0}\right)\right) \\
& >\tau_{2} \min \left\{\frac{a-\sum_{k=1}^{m-2} a_{k}}{a\left(t_{0}-t_{1}\right)+b}, \frac{c-\sum_{k=1}^{m-2} b_{k}}{c\left(\sigma\left(t_{2}\right)-t_{0}\right)+d}\right\}\left(\left|u_{1}^{\Delta}\left(t_{0}\right)\right|+\left|u_{2}^{\Delta}\left(t_{0}\right)\right|\right) \\
& =\left|u_{1}^{\Delta}\left(t_{0}\right)\right|+\left|u_{2}^{\Delta}\left(t_{0}\right)\right|,
\end{aligned}
$$

this is a contradiction. The proof is complete. $\square$

To prove our main results, we recommend notation

$$
\begin{aligned}
\lambda_{1}^{-}= & \left(a-\sum_{k=1}^{m-2} a_{k}\right)\left(c\left(\sigma\left(t_{2}\right)\right)^{2}+d\left(\sigma\left(t_{2}\right)+\sigma^{2}\left(t_{2}\right)\right)-\sum_{k=1}^{m-2} b_{k} \xi_{k}^{2}\right) \\
& +\left(c-\sum_{k=1}^{m-2} b_{k}\right)\left(\sum_{k=1}^{m-2} a_{k} \xi_{k}^{2}+b\left(t_{1}+\sigma\left(t_{1}\right)\right)-a t_{1}^{2}\right) \\
\lambda_{2}^{-}= & \left(\sum_{k=1}^{m-2} a_{k} \xi_{k}^{2}+b\left(t_{1}+\sigma\left(t_{1}\right)\right)-a t_{1}^{2}\right)\left(\sum_{k=1}^{m-2} b_{k} \xi_{k}-c \sigma\left(t_{2}\right)-d\right) \\
& +\left(c\left(\sigma\left(t_{2}\right)\right)^{2}+d\left(\sigma\left(t_{2}\right)+\sigma^{2}\left(t_{2}\right)\right)-\sum_{k=1}^{m-2} b_{k} \xi_{k}^{2}\right)\left(\sum_{k=1}^{m-2} a_{k} \xi_{k}+b-a t_{1}\right) \\
\bar{\lambda}= & \left(a-\sum_{k=1}^{m-2} a_{k}\right)\left(c \sigma\left(t_{2}\right)+d-\sum_{k=1}^{m-2} b_{k} \xi_{k}\right)+\left(c-\sum_{k=1}^{m-2} b_{k}\right)\left(\sum_{k=1}^{m-2} a_{k} \xi_{k}+b-a t_{1}\right), \\
\lambda_{1}= & \frac{\lambda_{1}^{-}}{\bar{\lambda}}, \quad \lambda_{2}=\frac{\lambda_{2}^{-}}{\bar{\lambda}}
\end{aligned}
$$

where $\sigma^{2}\left(t_{2}\right)=\sigma\left(\sigma\left(t_{2}\right)\right)$, and for $i=1,2$,

$$
\begin{gathered}
L_{i}=\max \left\{\left|x^{\Delta}\left(t_{1}\right) \int_{t_{1}}^{t_{2}} \frac{1}{r} \gamma(\sigma(s)) h_{i}(s) \Delta s+x^{\Delta}\left(t_{1}\right) A\left(h_{i}\right)+y^{\Delta}\left(t_{1}\right) B\left(h_{i}\right)\right|,\right. \\
\left.\left|y^{\Delta}\left(t_{2}\right) \int_{t_{1}}^{t_{2}} \frac{1}{r} x(\sigma(s)) h_{i}(s) \Delta s+x^{\Delta}\left(t_{2}\right) A\left(h_{i}\right)+y^{\Delta}\left(t_{2}\right) B\left(h_{i}\right)\right|\right\}, \\
M_{i}=\min \left\{\int_{t_{1}}^{t_{2}} G(\eta, s) h_{i}(s) \Delta s+x(\eta) A\left(h_{i}\right)+\gamma(\eta) B\left(h_{i}\right),\right. \\
\left.\int_{t_{1}}^{t_{2}} G(\sigma(\omega), s) h_{i}(s) \Delta s+x(\sigma(\omega)) A\left(h_{i}\right)+\gamma(\sigma(\omega)) B\left(h_{i}\right)\right\}, \\
N_{i}=\sup _{t \in\left[t_{1}, \sigma\left(t_{2}\right)\right]_{\mathbb{T}}}\left(\int_{t_{1}}^{t_{2}} G(t, s) h_{i}(s) \Delta s+x(t) A\left(h_{i}\right)+\gamma(t) B\left(h_{i}\right)\right) .
\end{gathered}
$$


Theorem 3.2 Suppose $\left(A_{1}\right)-\left(A_{3}\right)$ hold and $f_{i}(t, 0,0,0,0) \otimes 0(i=1,2)$ for $t \in\left[t_{1}, \sigma\left(t_{2}\right)\right]_{\mathbb{T}}$. Assume that there exist positive numbers $m_{1}, m_{2}$ and $m_{4}$ such that $m_{1}$ $<m_{2} \leq \frac{\tau_{1}^{2}\left(\lambda_{1}^{2}+4 \lambda_{2}\right)}{8\left|\sigma\left(t_{2}\right)+\sigma^{2}\left(t_{2}\right)-\lambda_{1}\right|} m_{4}$ and

$\left(C_{1}\right)$ if $t \in\left[t_{1}, \sigma\left(t_{2}\right)\right]_{\mathbb{T}}, v_{1}, v_{2} \geq 0, v_{1}+v_{2} \in\left[0, \tau_{2} m_{4}\right]$ and $w_{1}+w_{2} \in\left[-m_{4}, m_{4}\right]$, then $f_{i}\left(t, v_{1}, v_{2}, w_{1}, w_{2}\right) \leq \frac{m_{4}}{2 L_{i}} \quad(i=1,2)$;

$\left(C_{2}\right)$ there exists $i_{0} \in\{1,2\}$, such that

$$
f_{i_{0}}\left(t, v_{1}, v_{2}, w_{1}, w_{2}\right)>\frac{m_{2}}{2 M_{i_{0}}}
$$

for $t \in[\eta, \sigma(\omega)]_{\mathbb{T}}, v_{1}, v_{2} \geq 0, v_{1}+v_{2} \in\left[m_{2}, m_{2} \tau_{1}^{-2}\right]$ and $w_{1}+w_{2} \in\left[-m_{4}, m_{4}\right]$

$\left(C_{3}\right)$ if $t \in\left[t_{1}, \sigma\left(t_{2}\right)\right]_{\mathbb{T}}, v_{1}, v_{2} \geq 0, v_{1}+v_{2} \in\left[0, m_{1}\right]$ and $w_{1}+w_{2} \in\left[-m_{4}, m_{4}\right]$, then

$$
f_{i}\left(t, v_{1}, v_{2}, w_{1}, w_{2}\right) \leq \frac{m_{1}}{2 N_{i}} \quad(i=1,2) .
$$

Then the system $(S)$ has at least three positive solutions $u_{1}, u_{2}$, and $u_{3}$ satisfying

$$
\begin{gathered}
\gamma\left(u_{j}\right) \leq m_{4}(j=\mathbb{1}, 2, \Re) ; \\
m_{2}<\alpha\left(u_{\mathbb{1}}\right) ; \quad m_{1}<\beta\left(u_{2}\right) \text { with } \alpha\left(u_{2}\right)<m_{2} ; \quad \beta\left(u_{\Re}\right)<m_{1} .
\end{gathered}
$$

Proof. Define the operator $T$ by

$$
(T u)(t)=\left(\left(T_{1}\right)(t),\left(T_{2}\right)(t)\right),
$$

where $\quad\left(T_{i}\right)(t)=\int_{t_{1}}^{t_{2}} G(t, s) h_{i}(s) f_{i}\left(s, u_{1}(s), u_{2}(s), u_{1}^{\Delta}(s), u_{2}^{\Delta}(s)\right) \Delta s+x(t) A\left(h_{i} f_{i}\right)+y(t) B\left(h_{i} f_{i}\right)(i=1,2)$ for $t \in\left[t_{1}, \sigma\left(t_{2}\right)\right]_{\mathbb{T}}$. For $u=\left(u_{1}, u_{2}\right) \in P$, from Lemma 2.2 we note that the system $(S)$ has a solution $u$ if and only if $u$ is the fixed point of $u(t)=(T u)(t)$. Hypothesis $\left(A_{3}\right)$ implies that $A\left(h_{i} f_{i}\right) \geq 0, B\left(h_{i} f_{i}\right) \geq 0(i=1,2)$. By Equation 2.4, we have $\left(T_{i}\right)(t) \geq 0(i=$ 1 , 2) for $t \in\left[t_{1}, \sigma\left(t_{2}\right)\right]_{\mathbb{\pi}},\left(T_{i}\right)(t)(i=1,2)$ is concave on $\left[t_{1}, \sigma\left(t_{2}\right)\right]_{\mathbb{\pi}}$ and satisfies $c T_{i}\left(\sigma\left(t_{2}\right)\right)+d T_{i}^{\Delta}\left(\sigma\left(t_{2}\right)\right)=\sum_{k=1}^{m-2} b_{k} T_{i}\left(\xi_{k}\right), c T_{i}\left(\sigma\left(t_{2}\right)\right)+d T_{i}^{\Delta}\left(\sigma\left(t_{2}\right)\right)=\sum_{k=1}^{m-2} b_{k} T_{i}\left(\xi_{k}\right),(i=$ 1, 2) Further we can obtain that $\inf _{t \in[\eta, \sigma(\omega)]_{\top}} T_{1}(t)+\inf _{t \in[\eta, \sigma(\omega)]_{\mathbb{T}}} T_{2}(t) \geq \tau_{1}\left(\sup _{t \in\left[t_{1}, \sigma\left(t_{2}\right)\right]_{\mathbb{T}}}\left|T_{1}(t)\right|+\sup _{t \in\left[t_{1}, \sigma\left(t_{2}\right)\right]_{\mathbb{T}}}\left|T_{2}(t)\right|\right.$, since for $i$ $=1,2$,

$$
\begin{aligned}
& \sup _{t \in\left[t_{1}, \sigma\left(t_{2}\right)\right]_{\mathbb{T}}}\left|\left(T_{i}\right)(t)\right| \\
& =\sup _{t \in\left[t_{1}, \sigma\left(t_{2}\right)\right]_{\mathbb{T}}}\left(\int_{t_{1}}^{t_{2}} G(t, s) h_{i}(s) f_{i}\left(s, u_{1}(s), u_{2}(s), u_{1}^{\Delta}(s), u_{2}^{\Delta}(s)\right) \Delta s+x(t) A\left(h_{i} f_{i}\right)+y(t) B\left(h_{i} f_{i}\right)\right) \\
& \leq \int_{t_{1}}^{t_{2}} G(\sigma(s), s) h_{i}(s) f_{i}\left(s, u_{1}(s), u_{2}(s), u_{1}^{\Delta}(s), u_{2}^{\Delta}(s)\right) \Delta s+x\left(\sigma\left(t_{2}\right)\right) A\left(h_{i} f_{i}\right)+\gamma\left(t_{1}\right) B\left(h_{i} f_{i}\right),
\end{aligned}
$$

on the other hand, for $t \in[\eta, \sigma(\omega)]_{\mathbb{T}}$, Equation 2.2 implies that there exists $\tau_{1} \in(0$, 1) such that 


$$
\begin{aligned}
\left(T_{i}\right)(t)= & \int_{t_{1}}^{t_{2}} G(t, s) h_{i}(s) f_{i}\left(s, u_{1}(s), u_{2}(s), u_{1}^{\Delta}(s), u_{2}^{\Delta}(s)\right) \Delta s+x(t) A\left(h_{i} f_{i}\right)+\gamma(t) B\left(h_{i} f_{i}\right) \\
\geq & \int_{t_{1}}^{t_{2}} \tau_{1} G(\sigma(s), s) h_{i}(s) f_{i}\left(s, u_{1}(s), u_{2}(s), u_{1}^{\Delta}(s), u_{2}^{\Delta}(s)\right) \Delta s+x\left(\frac{3 t_{1}+\sigma\left(t_{2}\right)}{4}\right) A\left(h_{i} f_{i}\right) \\
& +\gamma\left(\frac{t_{1}+3 \sigma\left(t_{2}\right)}{4}\right) B\left(h_{i} f_{i}\right) \\
\geq & \left.\tau_{1}\left(\int_{t_{1}}^{t_{2}} G \sigma(s), s\right) h_{i}(s) f_{i}\left(s, u_{1}(s), u_{2}(s), u_{1}^{\Delta}(s), u_{2}^{\Delta}(s)\right) \Delta s+x\left(\sigma\left(t_{2}\right)\right) A\left(h_{i} f_{i}\right)+y\left(t_{1}\right) B\left(h_{i} f_{i}\right)\right) \\
\geq & \tau_{1} \sup _{t \in\left[t_{1}, \sigma\left(t_{2}\right)\right]_{\mathbb{T}}}\left|\left(T_{i}\right)(t)\right| .
\end{aligned}
$$

Thus, we can conclude that $T u \in P$. Furthermore, it is easy to check that the operator $T$ is completely continuous by using Arzela-Ascoli lemma, and so the conditions of Lemma 2.1 hold with respect to T. $\alpha, \gamma, \theta$, and $\beta$ are defined by Equation 3.2, we also note that $\alpha, \gamma, \theta, \beta: P \rightarrow[0, \infty)$ are continuous nonnegative functionals such that $\alpha(u)$ $\leq \theta(u)=\beta(u)$ for all $u \in P, \beta$ satisfies $\beta(\lambda u) \leq \lambda \beta(u)$ for $0 \leq \lambda \leq 1$, and $|u| \leq \varepsilon \gamma(u)$ for all $u \in \overline{P\left(\gamma, m_{4}\right)}$, where $\varepsilon=\max \left\{\tau_{2}, 1\right\}$. We now show that if assumption $\left(C_{1}\right)$ is satisfied, then

$$
\begin{aligned}
& T: \overline{P\left(\gamma, m_{4}\right)} \rightarrow \overline{P\left(\gamma, m_{4}\right)} \\
& \text { In fact, for } u=\left(u_{1}, u_{2}\right) \in \overline{P\left(\gamma, m_{4}\right)} \text {, we have } \\
& \gamma(u)=\sup _{t \in\left[t_{1}, t_{2}\right]_{\mathbb{T}}}\left|u_{1}^{\Delta}(t)\right|+\sup _{t \in\left[t_{1}, t_{2}\right]_{\mathbb{T}}}\left|u_{2}^{\Delta}(t)\right| \leq m_{4} \text {. By Lemma } 3.1 \text {, it holds } \\
& \sup _{t \in\left[t_{1}, \sigma\left(t_{2}\right)\right]_{\pi}}\left|u_{1}(t)\right|+\sup _{t \in\left[t_{1}, \sigma\left(t_{2}\right)\right]_{\pi}}\left|u_{2}(t)\right| \leq \tau_{2}\left(\sup _{t \in\left[t_{1}, t_{2}\right]_{\pi}}\left|u_{1}^{\Delta}(t)\right|+\sup _{t \in\left[t_{1}, t_{2}\right]_{\pi}}\left|u_{2}^{\Delta}(t)\right|\right) \leq \tau_{2} m_{4}, \quad \text { and } \quad \text { so } \\
& \text { assumption }\left(C_{1}\right) \text { implies } f_{i}\left(t, u_{1}(t), u_{2}(t), u_{1}^{\Delta}(t), u_{1}^{\Delta}(t)\right) \leq \frac{m_{4}}{2 L_{i}}(i=1,2) \text {, for } \\
& t \in\left[t_{1}, \sigma\left(t_{2}\right)\right]_{\mathbb{T}} \text {. On the other hand, for } u \in P \text {, we have proved that } T u=\left(T_{1}, T_{2}\right) \in P \text {, } \\
& \sup _{t \in\left[t_{1}, t_{2}\right]_{\mathbb{T}}}\left|\left(T_{i}\right)^{\Delta}(t)\right| \\
& =\sup _{t \in\left[t_{1}, t_{2}\right]_{\mathbb{T}}} \mid y^{\Delta}(t) \int_{t_{1}}^{t} \frac{1}{r} x(\sigma(s)) h_{i}(s) f_{i}\left(s, u_{1}(s), u_{2}(s), u_{1}^{\Delta}(s), u_{2}^{\Delta}(s)\right) \Delta s \\
& +x^{\Delta}(t) \int_{t}^{t_{2}} \frac{1}{r} y(\sigma(s)) h_{i}(s) f_{i}\left(s, u_{1}(s), u_{2}(s), u_{1}^{\Delta}(s), u_{2}^{\Delta}(s)\right) \Delta s+x^{\Delta}(t) A\left(h_{i} f_{i}\right) \\
& +y^{\Delta}(t) B\left(h_{i} f_{i}\right) \mid \\
& \leq \frac{m_{4}}{2 L_{i}} \max \left\{\left|x^{\Delta}\left(t_{1}\right) \int_{t_{1}}^{t_{2}} \frac{1}{r} \gamma(\sigma(s)) h_{i}(s) \Delta s+x^{\Delta}\left(t_{1}\right) A\left(h_{i}\right)+y^{\Delta}\left(t_{1}\right) B\left(h_{i}\right)\right|,\right. \\
& \left.\left|y^{\Delta}\left(t_{2}\right) \int_{t_{1}}^{t_{2}} \frac{1}{r} x(\sigma(s)) h_{i}(s) \Delta s+x^{\Delta}\left(t_{2}\right) A\left(h_{i}\right)+y^{\Delta}\left(t_{2}\right) B\left(h_{i}\right)\right|\right\}=\frac{m_{4}}{2} \quad(i=1,2) .
\end{aligned}
$$

Thus,

$$
\gamma(T u)=\sup _{t \in\left[t_{1}, t_{2}\right]_{\mathbb{T}}}\left|\left(T_{1}\right)^{\Delta}(t)\right|+\sup _{t \in\left[t_{1}, t_{2}\right]_{\mathbb{T}}}\left|\left(T_{2}\right)^{\Delta}(t)\right| \leq m_{4}
$$

Therefore, Equation 3.4 holds. 
To check condition $\left(B_{1}\right)$ in Lemma 2.1 , we choose $u_{0}=\left(u_{10}, u_{20}\right)$, taking $t \in\left[t_{1}, \sigma\left(t_{2}\right)\right]_{\mathbb{T}}, t \in\left[t_{1}, \sigma\left(t_{2}\right)\right]_{\mathbb{T}}$. where $\bar{m}=\frac{4 m_{2}}{\tau_{1}^{2}\left(\lambda_{1}^{2}+4 \lambda_{2}\right)}>0$. It is not difficult to verify that $u_{i 0}(t) \geq 0(i=1,2), u_{i 0}(t)(i=1,2)$ is concave on $\left[t_{1}, \sigma\left(t_{2}\right)\right]_{\mathbb{T}}$ and satisfies $a u_{i 0}\left(t_{1}\right)-b u_{i 0}^{\Delta}\left(t_{1}\right)=\sum_{k=1}^{m-2} a_{k} u_{i 0}\left(\xi_{k}\right), c u_{i 0}\left(\sigma\left(t_{2}\right)\right)+d u_{i 0}^{\Delta}\left(\sigma\left(t_{2}\right)\right)=\sum_{k=1}^{m-2} b_{k} u_{i 0}\left(\xi_{k}\right)(i=1,2)$. By the properties of $u_{i 0}(t)(i=1,2)$, we can obtain

$$
\begin{aligned}
\alpha\left(u_{0}\right) & =\inf _{t \in[\eta, \sigma(\omega)]_{\pi}}\left|u_{10}(t)\right|+\inf _{t \in[\eta, \sigma(\omega)]_{\pi}}\left|u_{20}(t)\right| \\
& =\min \left\{u_{10}(\eta), u_{10}(\sigma(\omega))\right\}+\min \left\{u_{20}(\eta), u_{20}(\sigma(\omega))\right\} \\
& \geq \tau_{1}\left(\frac{m_{2}}{\tau_{1}^{2}}+\frac{m_{2}}{\tau_{1}^{2}}\right)>m_{2}, \\
\theta\left(u_{0}\right) & =\sup _{t \in\left[t_{1}, \sigma\left(t_{2}\right)\right]_{\pi}}\left|u_{10}(t)\right|+\sup _{t \in\left[t_{1}, \sigma\left(t_{2}\right)\right]_{\top}}\left|u_{20}(t)\right|=u_{10}\left(\frac{\lambda_{1}}{2}\right)+u_{20}\left(\frac{\lambda_{1}}{2}\right)=\frac{m_{2}}{\tau_{1}^{2}}+\frac{m_{2}}{\tau_{1}^{2}}>m_{2}, \\
\gamma\left(u_{0}\right) & =\sup _{t \in\left[t_{1}, t_{2}\right]_{\pi}}\left|u_{10}^{\Delta}(t)\right|+\sup _{t \in\left[t_{1}, t_{2}\right]_{\pi}}\left|u_{20}^{\Delta}(t)\right| \\
& =\max \left\{\left|u_{10}^{\Delta}\left(t_{1}\right)\right|,\left|u_{10}^{\Delta}\left(\sigma\left(t_{2}\right)\right)\right|\right\}+\max \left\{\left|u_{20}^{\Delta}\left(t_{1}\right)\right|,\left|u_{20}^{\Delta}\left(\sigma\left(t_{2}\right)\right)\right|\right\} \leq m_{4} .
\end{aligned}
$$

$\begin{array}{lllll}\text { Equations } & 3.5 & \text { and } & 3.6 & \text { imply that }\end{array}$ $\inf _{t \in[\eta, \sigma(\omega)]_{\top}} u_{10}(t)+\inf _{t \in[\eta, \sigma(\omega)]_{\mathbb{T}}} u_{20}(t) \geq \tau_{1}\left(\sup _{t \in\left[t_{1}, \sigma\left(t_{2}\right)\right]_{\mathbb{T}}}\left|u_{10}(t)\right|+\sup _{t \in\left[t_{1}, \sigma\left(t_{2}\right)\right]_{\mathbb{T}}}\left|u_{20}(t)\right|\right.$, thus we get $u_{0} \in P$ and so $\alpha\left(u_{0}\right)>m_{2}, \theta\left(u_{0}\right)>m_{2}, \gamma\left(u_{0}\right) \leq m_{4}$ Further, $u_{0} \in P\left(\gamma, \theta, \alpha, m_{2}, m_{2} \tau_{1}^{-2}, m_{4}\right)$ and $\left\{u_{0} \in P\left(\gamma, \theta, \alpha, m_{2}, m_{2} \tau_{1}^{-2}, m_{4}\right): \alpha\left(u_{0}\right)>m_{2}\right\} \neq \emptyset$. For $\quad u \in P\left(\gamma, \theta, \alpha, m_{2}, m_{2} \tau_{1}^{-2}, m_{4}\right)$, there is $m_{2} \leq u_{1}(t)+u_{2}(t) \leq m_{2} \tau_{1}^{-2},\left|u_{1}^{\Delta}(t)+u_{2}^{\Delta}(t)\right| \leq m_{4}$ for $t \in[\eta, \sigma(\omega)]_{\mathbb{T}}$. Hence by assumption $\left(C_{2}\right)$, one has that $f_{i_{0}}\left(t, u_{1}(t), u_{2}(t), u_{1}^{\Delta}(t), u_{2}^{\Delta}(t)\right)>\frac{m_{2}}{2 M_{i_{0}}}\left(i_{0}=1,2\right)$ for $t \in[\eta, \sigma(\omega)]_{\mathbb{T}}$. So we obtain that, for $i_{0}=1,2$

$$
\begin{aligned}
\inf _{t \in[\eta, \sigma(\omega)]_{\pi}}\left|T_{i_{0}}(t)\right|=\min \left\{\left(T_{i_{0}}\right)(\eta),\left(T_{i_{0}}\right)(\sigma(\omega))\right\} \\
=\min \left\{\int_{t_{1}}^{t_{2}} G(\eta, s) h_{i_{0}}(s) f_{i_{0}}\left(s, u_{1}(s), u_{2}(s), u_{1}^{\Delta}(s), u_{2}^{\Delta}(s)\right) \Delta s+x(\eta) A\left(h_{i_{0}} f_{i_{0}}\right)\right. \\
\quad+\gamma(\eta) B\left(h_{i_{0}} f_{i_{0}}\right), \int_{t_{1}}^{t_{2}} G(\sigma(\omega), s) h_{i_{0}}(s) f_{i_{0}}\left(s, u_{1}(s), u_{2}(s), u_{1}^{\Delta}(s), u_{2}^{\Delta}(s)\right) \Delta s \\
\left.\quad+x(\sigma(\omega)) A\left(h_{i_{0}} f_{i_{0}}\right)+\gamma(\sigma(\omega)) B\left(h_{i_{0}} f_{i_{0}}\right)\right\} \\
\geq \frac{m_{2}}{2 M_{i_{0}}} \min \left\{\int_{t_{1}}^{t_{2}} G(\eta, s) h_{i_{0}}(s) \Delta s+x(\eta) A\left(h_{i_{0}}\right)+\gamma(\eta) B\left(h_{i_{0}}\right),\right. \\
\left.\quad \int_{t_{1}}^{t_{2}} G(\sigma(\omega), s) h_{i_{0}}(s) \Delta s+x(\sigma(\omega)) A\left(h_{i_{0}}\right)+y(\sigma(\omega)) B\left(h_{i_{0}}\right)\right\}=\frac{m_{2}}{2} .
\end{aligned}
$$

Thus,

$$
\alpha(T u)=\inf _{t \in[\eta, \sigma(\omega)]_{\mathbb{T}}}\left|T_{1}(t)\right|+\inf _{t \in[\eta, \sigma(\omega)]_{\mathbb{T}}}\left|T_{2}(t)\right|>m_{2} .
$$


So we get $\alpha(T u)>m_{2}$ for $u=\left(u_{1}, u_{2}\right) \in P\left(\gamma, \theta, \alpha, m_{2}, \frac{m_{2}}{\tau_{1}}, m_{4}\right)$ and condition $\left(B_{1}\right)$ in Lemma 2.1 is satisfied.

We now prove that condition $\left(B_{2}\right)$ in Lemma 2.1 holds. In fact, if $u=\left(u_{1}, u_{2}\right) \in P(\gamma$, $\left.\alpha, m_{2}, m_{4}\right)$ with $\theta(T u)>\frac{m_{2}}{\tau_{1}}$, then

$$
\alpha(T u) \geq \tau_{1} \theta(T u)>m_{2}
$$

Finally, we assert that condition $\left(B_{3}\right)$ in Lemma 2.1 also holds. $(0,0) \notin Q\left(\gamma, \beta, m_{1}\right.$, $\left.m_{4}\right)$, since $\beta((0,0))=0<m_{1}$. Assume that $u=\left(u_{1}, u_{2}\right) \in Q\left(\gamma, \beta, m_{1}, m_{4}\right)$ with $\beta(u)=$ $m_{1}$, assumption $\left(C_{3}\right)$ implies that

$$
\begin{aligned}
& \sup _{t \in\left[t_{1}, \sigma\left(t_{2}\right)\right]_{\mathbb{T}}}\left|T_{i}(t)\right| \\
& =\sup _{t \in\left[t_{1}, \sigma\left(t_{2}\right)\right]_{\mathbb{T}}} \int_{t_{1}}^{t_{2}} G(t, s) h_{i}(s) f_{i}\left(s, u_{1}(s), u_{2}(s), u_{1}^{\Delta}(s), u_{2}^{\Delta}(s)\right) \Delta s+x(t) A\left(h_{i} f_{i}\right)+\gamma(t) B\left(h_{i} f_{i}\right) \\
& \leq \frac{m_{1}}{2 N_{i}} \sup _{t \in\left[t_{1}, \sigma\left(t_{2}\right)\right]_{\mathbb{T}}}\left(\int_{t_{1}}^{t_{2}} G(t, s) h_{i}(s) \Delta s+x(t) A\left(h_{i}\right)+y(t) B\left(h_{i}\right)\right)=\frac{m_{1}}{2} \quad(i=1,2) .
\end{aligned}
$$

Hence,

$$
\beta(T u)=\sup _{t \in\left[t_{1}, \sigma\left(t_{2}\right)\right]_{\mathbb{T}}}\left|T_{1}(t)\right|+\sup _{t \in\left[t_{1}, \sigma\left(t_{2}\right)\right]_{\mathbb{T}}}\left|T_{2}(t)\right| \leq m_{1} .
$$

Therefore, the conditions of Lemma 2.1 are fulfillled, thus the system $(S)$ has at least three positive solutions $u_{1}, u_{2}$, and $u_{3}$, such that Equation 3.3 hold. $\square$

From the above argument, we could consider naturally the general dynamic system

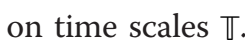

$$
\left\{\begin{array}{l}
u_{1}^{\Delta \Delta}(t)+h_{1}(t) f_{1}\left(t, u_{1}(t), \ldots, u_{n}(t), u_{1}^{\Delta}(t), \ldots, u_{n}^{\Delta}(t)\right)=0, \\
\ldots, \\
u_{n}^{\Delta \Delta}(t)+h_{n}(t) f_{n}\left(t, u_{1}(t), \ldots, u_{n}(t), u_{1}^{\Delta}(t), \ldots, u_{n}^{\Delta}(t)\right)=0 \\
a u_{i}\left(t_{1}\right)-b u_{i}^{\Delta}\left(t_{1}\right)=\sum_{k=1}^{m-2} a_{k} u_{i}\left(\xi_{k}\right), \quad(i=1,2, \ldots, n) \\
c u_{i}\left(\sigma\left(t_{2}\right)\right)+d u_{i}^{\Delta}\left(\sigma\left(t_{2}\right)\right)=\sum_{k=1}^{m-2} b_{k} u_{i}\left(\xi_{k}\right),
\end{array}(i=1,\right.
$$

where $a, b, c, d, a_{k}, b_{k}, \xi_{k}(k=1,2, \ldots, m-2)$ satisfy $\left(A_{1}\right)$ and $h_{i}, f_{i}(i=1,2)$ fulfill the condition

$\left(A_{2}^{\prime}\right) h_{i} \in C_{r d}\left(\left[t_{1}, t_{2}\right]_{\pi},[0, \infty)\right)(i=1,2, \ldots, n)$ and there exists $t_{0} \in\left(t_{1}, t_{1}\right)_{\pi}$ such that $h_{i}\left(t_{0}\right)>0, f_{i} \in C\left(\left[t_{1}, t_{2}\right]_{\mathbb{\pi}} \times[0, \infty)^{n} \times(-\infty, \infty)^{n},[0, \infty)\right)(i=1,2, \ldots, n)$.

We obtain a result, the proof of the result is omitted.

Theorem 3.3 Suppose $\left(A_{1}\right),\left(A_{2}^{\prime}\right)$ and $\left(A_{3}\right)$ hold, and $f_{i}(t, 0, \ldots, 0) \otimes 0(i=1,2, \ldots, n)$ for $t \in\left[t_{1}, \sigma\left(t_{2}\right)\right]_{\mathbb{T}}$. Assume that there exist positive numbers $m_{1}^{\prime}, m_{2}^{\prime}, m_{4}^{\prime}, \tau_{1}^{\prime}, \tau_{2}^{\prime}$ such that $m_{1}^{\prime}<m_{2}^{\prime} \leq \frac{\left(\tau_{1}^{\prime}\right)^{2}\left(\lambda_{1}^{2}+4 \lambda_{2}\right)}{4 n\left|\sigma\left(t_{2}\right)+\sigma^{2}\left(t_{2}\right)-\lambda_{1}\right|} m_{4}^{\prime}, 0<\tau_{1}^{\prime}, \tau_{2}^{\prime}<1$ and

$\left(C_{4}\right) \quad$ if $\quad t \in\left[t_{1}, \sigma\left(t_{2}\right)\right]_{\mathbb{T}}, \quad v_{1}, \quad v_{2}, \quad \ldots, \quad v_{n} \geq 0, \quad v_{1}+v_{2}+\cdots+v_{n} \in\left[-\tau_{2}^{\prime}, m_{4}^{\prime}\right]$ and $w_{1}+w_{2}+\cdots w_{n} \in\left[-m_{4}^{\prime}, m_{4}^{\prime}\right]$, then

$$
f_{i}\left(t, v_{1}, \ldots, v_{n}, w_{1}, \ldots, w_{n}\right) \leq \frac{m_{4}^{\prime}}{n L_{i}^{\prime}} \quad(i=1,2, \ldots, n) ;
$$


$\left(C_{5}\right)$ there exists $i_{0} \in\{1,2, \ldots, n\}$, such that

$$
f_{i_{0}}\left(t, v_{1}, \ldots, v_{n}, w_{1}, \ldots, w_{n}\right)>\frac{m_{2}^{\prime}}{n M_{i_{0}}^{\prime}}
$$

for $t \in[\eta, \sigma(\omega)]_{\mathbb{T}}, \quad v_{1}, \quad v_{2}, \quad \ldots, \quad v_{n} \geq 0, \quad v_{1}+v_{2}+\cdots+v_{n} \in\left[m_{2}^{\prime}, m_{2}^{\prime}\left(\tau_{1}^{\prime}\right)^{-2}\right]$ and $w_{1}+w_{2}+\cdots w_{n} \in\left[-m_{4}^{\prime}, m_{4}^{\prime}\right]$

$\left(C_{6}\right) \quad$ if $t \in\left[t_{1}, \sigma\left(t_{2}\right)\right] \pi, \quad v_{1}, \quad v_{2}, \ldots, \quad v_{n} \geq 0, \quad v_{1}+v_{2}+\cdots+v_{n} \in\left[0, m_{1}^{\prime}\right]$ and $w_{1}+w_{2}+\cdots w_{n} \in\left[-m_{4}^{\prime}, m_{4}^{\prime}\right]$, then

$$
f_{i}\left(t, v_{1}, \ldots, v_{n}, w_{1}, \ldots, w_{n}\right) \leq \frac{m_{1}^{\prime}}{n N_{i}^{\prime}} \quad(i=1,2, \ldots, n)
$$

where $L_{1}^{\prime}, M_{i_{0}}^{\prime}, N_{i}^{\prime}\left(i, i_{0}=1,2, \ldots, n\right)$ are positive constants and are similar to $L_{i}, M_{i_{0}}, N_{i}$ $\left(i, i_{0}=1,2, \ldots, n\right)$, respectively.

Then the system (GS) has at least three positive solutions $u_{\mathbb{1}}, u_{\mathfrak{2}}$, and $u_{3 s a t i s f y i n g}$

$$
\begin{gathered}
\gamma\left(u_{j}\right) \leq m_{4}(j=\mathbb{1}, 2, \mathfrak{B}) ; \\
m_{2}<\alpha\left(u_{\mathbb{1}}\right) ; \quad m_{1}<\beta\left(u_{\mathfrak{2}}\right) \text { with } \alpha\left(u_{\mathfrak{2}}\right)<m_{2} ; \quad \beta\left(u_{\mathfrak{乃}}\right)<m_{1} .
\end{gathered}
$$

Let $\mathbb{T}=\{0\} \cup\left\{\frac{1}{2^{n+1}}\right\} \cup\left[\frac{1}{2}, 1\right](n=1,2, \ldots), h_{i}(t)=1,(i=1,2), m=4$. Consider the system

$$
\left\{\begin{array}{l}
u_{1}^{\Delta \Delta}(t)+f_{1}\left(t, u_{1}(t), u_{2}(t), u_{1}^{\Delta}(t), u_{2}^{\Delta}(t)\right)=0, \\
u_{2}^{\Delta \Delta}(t)+f_{2}\left(t, u_{1}(t), u_{2}(t), u_{1}^{\Delta}(t), u_{2}^{\Delta}(t)\right)=0, \\
4 u_{i}(0)-2 u_{i}^{\Delta}(0)=u_{i}\left(\frac{1}{4}\right)+u_{i}\left(\frac{1}{2}\right), \\
4 u_{i}(\sigma(1))+2 u_{i}^{\Delta}(\sigma(1))=u_{i}\left(\frac{1}{4}\right)+u_{i}\left(\frac{1}{2}\right),
\end{array}(i=1,2),\right.
$$

where

$$
f_{i}\left(t, v_{1}, v_{2}, w_{1}, w_{2}\right)=\left\{\begin{array}{c}
10^{-4} t+5\left(v_{1}+v_{2}\right)^{8}+10^{-3}\left(\frac{w_{1}+w_{2}}{2.510^{5}}\right)^{3}, v_{1}+v_{2}<4 \\
10^{-4} t+327680+10^{-3}\left(\frac{w_{1}+w_{2}}{2.5 \times 10^{5}}\right)^{3}, \quad v_{1}+v_{2} \geq 4
\end{array} \quad(i=1,2)\right.
$$

It is easy to check that hypotheses $\left(A_{1}\right)-\left(A_{3}\right)$ hold and $\mathrm{f}(\mathrm{t}, 0,0,0,0) \otimes 0$ on $[0,1]_{\mathbb{T}}$. By some calculations, we have $\tau_{1}=\frac{1}{2}, \tau_{2}=\frac{3}{2}, \lambda_{1}=1, \lambda_{2}=\frac{37}{32} L_{1}+L_{2}=\frac{25}{48}, M_{1}+M_{2} \approx \frac{31}{3}$, $N_{1}+N_{2}=\frac{104}{7}$. If we choose $m_{1}=\frac{1}{2}, m_{2}=1, m_{4}=2.5 \times 10^{5}$, then $f_{i}\left(t, v_{1}, v_{2}, w_{1}, w_{2}\right) \leq \frac{m_{4}}{2 L_{i}}=4.8 \times 10^{2}(i=1,2)$ for $t \in[0,1]_{\mathbb{T}}, v_{1}+v_{2} \in=\left[0, \frac{75}{2}\right], w_{1}+$ $w_{2} \in\left[-2.5 \times 10^{5}, 2.5 \times 10^{5}\right] ; f_{i_{0}}\left(t, v_{1}, v_{2}, w_{1}, w_{2}\right)>\frac{m_{2}}{M_{i_{0}}} \approx 0.09\left(i_{0} \in\{1,2\}\right)$ for

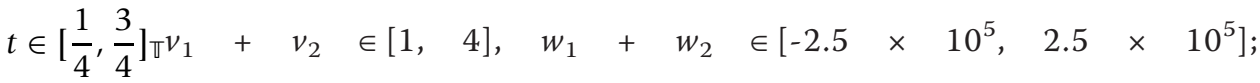
$f_{i}\left(t, v_{1}, v_{2}, w_{1}, w_{2}\right) \leq \frac{m_{1}}{2 N_{i}} \approx 0.03(i=1,2)$ for $t \in[0,1]_{\mathbb{T}}, v_{1}+v_{2} \in\left[0, \frac{1}{2}\right], w_{1}+w_{2} \in$ $\left[-2.5 \times 10^{5}, 2.5 \times 10^{5}\right]$. Thus all hypotheses of Theorem 3.2 hold. So the system (3.7) has at least three positive solutions $u_{\mathbb{1}}(t), u_{\mathbb{P}}(t)$ and $u_{\mathfrak{S}}(t)$ such that 
$\max _{0 \leq t \leq 1} u_{\Im}(t)<\frac{1}{2}$ with $\min _{\frac{1}{4} \leq t \leq \frac{3}{4}} u_{\mathcal{2}}(t)<1, \max _{0 \leq t \leq 1} u_{\Im}(t)<\frac{1}{2}$ hold.

\section{Competing interests}

The author declares that they have no competing interests.

Received: 22 December 2010 Accepted: 15 August 2011 Published: 15 August 2011

\section{References}

1. Bohner, M, Peterson, A: Dynamic Equations on Time Scales: An Introduction with Applications. Birkhäuser, Boston. (2001)

2. Bohner, M, Peterson, A: Advances in Dynamic Equations on Time Scales. Birkhäusere, Boston. (2003)

3. Jones, MA, Song, B, Thomas, DM: Controlling wound healing through debridement. Math Comput Modelling. 40 1057-1064 (2004). doi:10.1016/.mcm.2003.09.041

4. Atici, FM, Biles, DC, Lebedinsky, A: An appilcation of time scales to economics. Math Comput Model. 43, 718-726 (2006). doi:10.1016/.mcm.2005.08.014

5. Tisdell, C, Zaidi, A: Basic qualitative and quantitative results for solutions to nonlinear, dynamic equations on time scales with an application to economic modelling. Nonlinear Anal. 68, 3504-3524 (2008). doi:10.1016/j.na.2007.03.043

6. Wang, H: On the number of positive solutions of nonlinear systems. J Math Anal Appl. 281, 287-306 (2003)

7. Zhang, X, Liu, L: A necessary and sufficient condition of existence of positive solutions for nonlinear singular differential systems. J Math Anal Appl. 327, 400-414 (2007). doi:10.1016/j.jmaa.2006.04.031

8. Agarwal, RP, O'Regan, D, Wong, PJY: Positive Solutions of Differential, Difference and Integral Equations. Kluwer, Dordrecht. (1999)

9. Li, W, Sun, H: Multiple positive solutions for nonlinear dynamical systems on a measure chain. J Comput Appl Math. 162, 421-430 (2004). doi:10.1016/i.cam.2003.08.032

10. Choi, S, Im, D, Koo, N: Stability of linear dynamic systems on time scales. Adv Differ Equ. 670203 (2008)

11. Sun, J, Zhao, Y, Li, W: Existence of positive solution for second-order nonlinear discrete system with parameter. Math Comput Model. 41, 493-499 (2005). doi:10.1016/j.mcm.2004.04.006

12. Shao, N, Zhang, Y: Existence of positive solutions for semipositone dynamic system on time scales. Electron J Differ Equ. 114, 1-10 (2008)

13. Atici, FM, Guseinov, GSh: On Green's functions and positive solutions for boundary value problem on time scales. J Comput Appl Math. 141, 75-99 (2002). doi:10.1016/\$0377-0427(01)00437-X

14. Zhang, Y: A multiplicity result for a generalized Sturm-Liouville problem on time scales. J Differ Equ Appl. 16, 963-974 (2010). doi:10.1080/10236190802660476

15. Avery, RI, Peterson, AC: Three positive fixed points of nonlinear operators on ordered Banach spaces. Comput Math Appl. 42, 313-322 (2001). doi:10.1016/50898-1221(01)00156-0

16. Erbe, L, Peterson, A: Positive solutions for a nonlinear differential equation on a measure chain. Boundary Value Problems and Related Topics. Math Comput Modelling. 32(5-6), 571-585 (2000)

17. $\mathrm{Ma}$, R: Multiple positive solutions for nonlinear m-point boundary value problem. Appl Math Comput. 148, 249-262 (2004). doi:10.1016/50096-3003(02)00843-3

18. Zhang, Y, Sun, H: Three positive solutions for a generalized Sturm-Liouville multipoint BVP with dependence on the first order derivative. Dyn Syst Appl. 17, 313-3240 (2008)

doi:10.1186/1687-1847-2011-24

Cite this article as: Zhang: Multiplicity results for a generalized Sturm-Liouville dynamical system on time scales. Advances in Difference Equations 2011 2011:24.

\section{Submit your manuscript to a SpringerOpen ${ }^{\circ}$ journal and benefit from:}

- Convenient online submission

- Rigorous peer review

- Immediate publication on acceptance

- Open access: articles freely available online

- High visibility within the field

- Retaining the copyright to your article

Submit your next manuscript at $\gg$ springeropen.com 